\title{
Comparisons of the active components in four unripe raspberry extracts and their activites
}

\author{
Jing YANG ${ }^{1,2 *}\left(\mathbb{D}\right.$, Jingyan CUI ${ }^{1}$, Yanhui WU ${ }^{1}$, Hongjuan $\mathrm{HAN}^{1}$, Jinxiang $\mathrm{CHEN}^{1}$, Jingyang YAO ${ }^{1}$, Yongping LIU $^{1}$
}

\begin{abstract}
Dried unripe raspberry of Rubus chingii, were prepared as the following four extracts: unpurified raspberry extract (URE), purified raspberry extract (PRE), URE after high temperature-high pressure (UPE-H, HTHP), and PRE after HTHP (PRE-H). The total polyphenolic contents (TPCs) and total flavonoid contents (TFCs) of the four extracts, as assessed by colorimetry, significantly increased in the following order: URE $<$ URE-H $<$ PRE $<$ PRE-H. The antioxidant capacity and antibacterial activity were as follows: PRE-H $>$ PRE $\approx$ URE-H $>$ URE. Moreover, 11 typical polyphenols were quantified using a HPLC-MS analysis showed that HTHP treatments were conducive to releasing more (+)-catechin, (-)-epicatechin, proanthocyanidin B1 and ellagic acid, reaching $\sim 70 \%-1,100 \%$, but decreased the rutin, quercetin-3-O-glucuronide, kaempferol-3-O-rutinoside and kaempferol-3-O-glucoside levels by $\sim 10 \%-20 \%$. The purification of macroporous resin was beneficial to the enrichment of all polyphenols, by $\sim 30 \%-300 \%$. Ellagic acid was the main polyphenol found in the extracts, accounting for $\sim 67 \%-74 \%$. The study indicated that changes in the polyphenols of raspberry extracts after resin purification and/or HTHP treatment were correlated with the extract's antioxidant capacity and antibacterial activity. Further evaluations of the correlation between bioactive ingredients and treatments should reveal that HTHP and resin purification methods are effective means of preparing extracts.
\end{abstract}

Keywords: raspberry extracts; polyphenols; antioxidant capacity; antibacterial activity.

Practical Application: Unripe raspberry extracts can be processed into commercially promising high-polyphenol products.

\section{Introduction}

Berries, owing to the high polyphenol contents, are a good source of natural antioxidants. The total polyphenolic contents (TPCs) of cloudberries, raspberries and strawberries are the highest among eight tested berry extracts (Puupponen-Pimiä et al., 2001). The ripe raspberry of Rubus chingii $\mathrm{Hu}$, including Rubus idaeus, Rubus occidental, and Rubus species, is a nutritious fruit with various bioactivities, mainly associated with anthocyanins and tannins (Oszmianski et al., 2015; Sójka et al., 2016). The unripe raspberry of $R$. chingii, having more polyphenols (e.g., ellagic acid and tannin), is the only medicinal herbal included in The Chinese Pharmacopoeia. To balance on- and off-years and avoid unstable fruit yields, the plantations regularly remove flowers and young fruit (Goldschmidt, 2013). The unripe fruit are higher in different bioactive components, such as chlorogenic acid and gallic acid, than the ripe berries, which are rich in anthocyanins (Silva et al., 2017; Zhang et al., 2015b). Unripe raspberry from medicinal materials or plantation waste are not suitable as fresh food, but their extracts can be purified and processed into commercially promising high polyphenol products for use in pharmaceuticals and as food additives.

The bioactivity levels of the extracts vary greatly depending on the components and structures of the compounds in the extracts themselves, as well as the extraction conditions. For example, the low solubility levels of flavonoid aglycones (quercetin and kaempferol) in water often makes medicinal applications problematic, but they are highly soluble in methanol, ethanol, ethyl acetate and other organic solvents (Daglia, 2012; Kumar \& Pandey, 2013). In addition, tannins, polymerized polyphenols, can be roughly classified into two groups: 1) hydrolysable tannins (gallotannins and ellagitannins), and 2) condensed tannins (proanthocyanidins, PAs), oligomers $(<5)$ or polymers $(>5)$ of flavan-3-ol units, such as catechin or its derivative gallocatechin, are formed through the polymerization of carbon-carbon bonds between units. The polymers may escape analysis owing to their large molecular weights, which can exceed the limited MS scanning range $(\mathrm{m} / z: 100-2,000)$, and reduce their medicinal values because of their solubility levels (Shay et al., 2017). Boiling, frying, and high temperature and/or high pressure (HTHP) treatments of food in daily life may be beneficial to the depolymerization and/or hydrolyzation of these tannins. The TPCs and total flavonoids contents (TFCs) of foods increase during processing and are related to the higher antioxidant capacity (AC) and antibacterial activity levels (Gornas et al., 2016).

The raw extracts always contain chlorophyll, proteins, polysaccharides, saponin and other impurities (Zhang et al., 2015a; Zhang et al., 2015b), which limit their applications. Macroporous resins, such as XDA-6 and AB-8, are weak polar resins that have been widely used in the separation and purification of plant polyphenols through electrostatic force, hydrogen bonding interactions, complexation and size sieving (Gao et al., 2018; Xi et al., 
2015). Here, four kinds of raspberry extracts were produced using different methods, as follows: 1) Unpurified raspberry extract (URE) was prepared from dried unripe raspberry powder by ultrasonic-assistant ethanol method; 2) URE-H was prepared from URE by HTHP; 3) purified raspberry extract (PRE) was prepared from URE by XDA-6 macroporous resin purification; and 4) PRE-H was prepared from PRE by HTHP. This study investigated the TPCs, TFCs, ACs and antibacterial activity levels of the four raspberry extracts. The changes in 11 typical polyphenols (Supplementary Material Figure S1) found in the four extracts were analyzed by quantitative HPLC-MS. These results increase our understanding of the effects of different preparatory methods on the bioactive ingredients in raspberry extracts. The increased bioactive ingredients (e.g., polyphenols and flavonoids) may induce the higher AC and antibacterial activity level. The efficient preparation of high purity extracts has great industrial production potential.

\section{Materials and methods}

\subsection{Reagents}

(+)-Catechin (C), (-)-epicatechin (EC), brevifolin carboxylic acid (BCA), ellagic acid (EA), proanthocyanidin B1 (B1), quercetin (Q), quercetin-3-O-rutinoside (rutin), quercetin-3-O-glucuronide (Q3GA), kaempferol (K), kaempferol-3-O-rutinoside (K3R) and kaempferol-3-O-glucoside (K3G) were purchased from Shanghai Yuanye Bio-Technology Co., Ltd.(China). Yeast extract, peptone (Oxoid, USA), gallic acid, Folin-Ciocalteu's phenol, dimethyl sulphoxide (DMSO), 1,1-diphenyl-2-picryl-hydrazyl (DPPH) and 2, 2-azino-bis (ABTS) (Solarbio, China) were used in this study. LC/MS grade methanol, ethanol, hydrochloric acid, acetic acid and other reagents were purchased from Thermo Fisher Scientific (USA).

\subsection{Raspberry extracts}

Dried unripe raspberry $(R$. chingii $\mathrm{Hu})$ was purchased in a local Pharmacy (Taiyuan, China). In short, the ripening process of red raspberry includes three stages: green, white and red. The unripe raspberry in this paper mainly refers to the green fruit stage. The ground dry raspberry formed a powder that was extracted using the ultrasonic-assisted ethanol method, in which $10 \mathrm{mg} / \mathrm{mL}$ of powder was extracted in $52 \%$ ethanol at $360 \mathrm{~W}$ for $45 \mathrm{~min}$, twice, using an Ultrasonic apparatus (JOYN-15AL,Shanghaiqiaoyue Co. China). The extraction solution was concentrated to contain no alcohol (or $<5 \%$ ) by vacuum rotary steam. The concentrated solution was freeze-dried into a brown powder, (1) the unpurified raspberry extract (URE). (2) The concentrated URE solution was purified by XDA-6 macroporous resin under the following purification conditions: six bed volumes (BVs)/h as the velocity of adsorption, $60 \%$ ethanol as the elution reagent, with four BV/h as the elution velocity and five BV effluent. The purified solution was concentrated and then freeze-dried into a light brown powder, the PRE. Additionally, the concentrated URE and PRE solutions were subjected to HTHP treatments $\left(121^{\circ} \mathrm{C}\right.$ and $0.2 \mathrm{MPa}$ for $\left.30 \mathrm{~min}\right)$ using an autoclave (YX-280, Shanghaiqiaoyue Co., China) and freeze-dried into powders, (3) URE-H and (4) PRE-H, respectively. These extracts were dissolved in less than 1\% DMSO.

\subsection{Bacteria and culture conditions}

Escherichia coli (CGMCC1.12883), Staphylococcus aureus (CGMCC1.282), and Salmonella enteric (CGMCC1.755) from China General Microbiological Culture Collection Center (CGMCC) were incubated with shaking in $\mathrm{LB}$ medium at $37^{\circ} \mathrm{C}$ incubator.

\subsection{Total polyphenolic and flavonoids contents}

TPC was determined using the Folin-Ciocalteu assay (Meda et al., 2005) and calculated by the following equation based on the gallic acid standard curve: $\mathrm{A}=11.331 \mathrm{C}+0.015$ $\left(r^{2}=0.999\right)$; where A was the absorbance, and $C$ was the concentration of gallic acid $(\mathrm{mg} / \mathrm{mL})$. TPC $/(\mathrm{mg} / \mathrm{g})=\mathrm{CV} / \mathrm{m}$; $\mathrm{C}$ was solution concentration $(\mathrm{mg} / \mathrm{mL}) ; \mathrm{V}$ was solution volume $(\mathrm{mL})$; $\mathrm{m}$ was the PRE weight $(\mathrm{g})$. TFC was determined by the aluminum chloride calorimetric method (Meda et al., 2005). TFC was calculated by the following equation based on the rutin standard curve: $A=10.696 C+0.0109\left(r^{2}=0.999\right)$; where A was the absorbance, and $\mathrm{C}$ was the concentration of rutin $(\mathrm{mg} / \mathrm{mL})$. $\mathrm{TFC} /(\mathrm{mg} / \mathrm{g})=\mathrm{nCV} / \mathrm{m}$, where $\mathrm{n}$ was diluted multiples; $\mathrm{C}$ was solution concentration $(\mathrm{mg} / \mathrm{mL})$; V was solution volume $(\mathrm{mL})$; $\mathrm{m}$ was the PRE (g).

\subsection{Antioxidant Capacity (AC)}

The ACs of the extracts were determined using the DPPH and ABTS tests according to previous methods (Meda et al., 2005; Oszmianski et al., 2015) with slight modifications. All of the tested samples were serially diluted (from 0.5 to $64 \mu \mathrm{g} / \mathrm{mL}$ ). The absorbance of the mixtures was measured at $517 \mathrm{~nm}(\mathrm{DPPH})$ and at $734 \mathrm{~nm}$ (ABTS) using the spectrophotometer. The DPPH/ ABTS capacity of the samples was calculated as: DPPH/ABTS radical scavenging rate $(\%)=\left[1-\left(\mathrm{A}_{\text {sample }}-\mathrm{A}_{\text {blank }}\right) /\left(\mathrm{A}_{\text {control }}\right)\right] \times 100$, where $\mathrm{A}_{\text {sample }}$ represents the DPPH/ABTS radical with sample solution; $\mathrm{A}_{\text {blank }}$ represents all reagents except DPPH/ABTS working solution; and $\mathrm{A}_{\text {control }}$ represents all reagents except the sample solution. The radical scavenging activity of samples was determined by the $\mathrm{IC}_{50}$ value. The $\mathrm{IC}_{50}$ value was the concentration of samples at which DPPH or ABTS radicals was scavenged by $50 \%$ based on above the determination and calculation of linear regression curve.

\subsection{Minimal Inhibitory Concentration (MIC)}

MICs of four kinds of extracts against the three bacteria were determined by the checkerboard method, respectively. An extract stock solution was freshly prepared by dissolving $64 \mathrm{mg}$ (or $40 \mathrm{mg}$ ) of the extract powder in $<1 \%$ of DMSO solution and were filtered through a $0.45 \mu \mathrm{m}$ filter membrane. All samples bacteria suspensions were diluted in fresh LB to adjust to an $\mathrm{OD}_{660}$ of 0.2 . Equal volumes $(100 \mu \mathrm{L})$ of bacteria and serial two-fold dilutions of extract solution or antibiotic solution in LB medium were mixed into the wells of a 96-well microplate. Wells with no bacteria or no compounds were the negative controls. After an incubation with shaking (50 rpm) of $18-24 \mathrm{~h}$ at $37^{\circ} \mathrm{C}$ incubator, bacterial growth was recorded visually. MICs were determined as the minimum concentrations at which no bacterial growth was observed. 


\subsection{HPLC-MS analysis}

HPLC-MS analyses were performed on a Thermo Scientific ${ }^{\mathrm{TM}}$ Exactive plus/Q Exactive HPLC-MS system (Thermo Fisher Scientific Inc, USA). Samples were performed with a Thermo Hypersil GOLD C ${ }_{18}$ column $(100 \mathrm{~mm} \times 2.1 \mathrm{~mm}, 3-\mu \mathrm{m}$ particle size, Thermo Fisher Scientific Inc). A gradient program was divided (phase A water $+0.1 \%$ formic acid) and phase B (acetonitrile). The flow rate was $0.3 \mathrm{~mL} / \mathrm{min}$, and the phase gradient was as follows: $10 \% \mathrm{~B}$ at $0-1.5 \mathrm{~min} ; 10 \%-25 \% \mathrm{~B}$ at $1.5-8 \mathrm{~min} ; 25 \%-70 \%$ at $8-9 \mathrm{~min} ; 70 \%-10 \%$ at $9-10.5 \mathrm{~min}$; and $10 \% \mathrm{~B}$ at $10.5-12 \mathrm{~min}$. The effluents were directed into the MS via the electrospray interface. Nitrogen was used as the nebulizing and drying gas. The detector (mass spectrum) operated under the following conditions: electrospray ionization (ESI) interface operating in negative mode; source: $3.5 \mathrm{kV}$; sheath gas flow rate: 40 , auxiliary gas flow: 15 ; capillary temperature: $320^{\circ} \mathrm{C}$ and heater temperature: $300^{\circ} \mathrm{C}$. HPLC-MS was recorded over a mass-to-charge $(\mathrm{m} / \mathrm{z})$ range of 100 to 1,000 .

\subsection{Statistical analysis}

The data are presented as means \pm standard deviations. A one way analyses of variance was performed using IBM SPSS Statistics 19.0, and $p$-values $<0.05$ were considered as significant.

\section{Results and discussion}

\subsection{Changes in the TPCs and TFCs of the four extracts}

Plant polyphenols, classified into flavonoids and nonflavonoids based on chemical structure, are ubiquitous secondary metabolites found in all plants (Daglia, 2012). In this study, the TPCs and TFCs of the extracts were significantly different $(p<0.05)$ and increased in the following order: $\mathrm{URE}<\mathrm{URE}-\mathrm{H}<\mathrm{PRE}<\mathrm{PRE}-\mathrm{H}$ (Figure 1). The TPC of the PRE-H increased 1.9-fold from $233.6 \mathrm{mg} / \mathrm{g}$ dry weight to $443.7 \mathrm{mg} / \mathrm{g}$ dry weight. Therefore, the purification and HTHP of the raw extract were conducive to enriching and releasing more polyphenols and flavonoids.

\subsection{Changes in the ACs and antibacterial activities of the four extracts}

The free radical scavenging activity is mediated by an electron donor molecule (antioxidant). Polyphenols can be used as hydrogen donors, reductants, and singlet oxygen quenchers, thus playing key anti-oxidation roles. The ACs of extracts can be assessed by $\mathrm{IC}_{50}$ values. A lower $\mathrm{IC}_{50}$ value indicated a higher $\mathrm{AC}$. The ACs of the URE $\left(\mathrm{IC}_{50 / \mathrm{DPPH}}=22.55 \mu \mathrm{g} / \mathrm{mL}\right.$ and $\left.\mathrm{IC}_{50 / \mathrm{ABTS}}=14.07 \mu \mathrm{g} / \mathrm{mL}\right)$ and PRE-H $\left(\mathrm{IC}_{50 / \mathrm{DPPH}}=9.29 \mu \mathrm{g} / \mathrm{mL}\right.$ and $\left.\mathrm{IC}_{50 / \mathrm{ABTS}}=4.31 \mu \mathrm{g} / \mathrm{mL}\right)$ were the lowest and the highest, respectively, but those of the URE-H and PRE were not statistically significantly different $(p>0.05)$ (Figure 2). The $\mathrm{IC}_{50}$ values of our extracts were much lower than those of many other plant extracts (Benchennouf et al., 2017). The extracts, in particular PRE-H, have good antioxidant potentials.

The bacteriostasis experiment produced a similar result. The lower the MIC dose results of the greater the bacteriostatic effect of the substance. The PRE-H, when tested against E. coli, S. aureus and S. enterica, showed the greatest antibacterial activities compared with the other extracts using the checkerboard and plate count method. The MICs of the PRE-H against the three tested bacteria were the lowest, at 2, 4 and $8 \mathrm{mg} / \mathrm{mL}$ (Table 1 and Figure S2), compared with those of the URE, URE-H and PRE (Table 1). The MICs of the URE-H and PRE against E. coli and $S$. aureus were the same, at 4 and $8 \mathrm{mg} / \mathrm{mL}$, respectively. In short, the ACs and antibacterial activities of the four extracts had the following order PRE-H $>\mathrm{PRE} \approx \mathrm{URE}-\mathrm{H}>\mathrm{URE}$. The increases in the AC and antioxidant activity were related to the increases in the polyphenol levels that resulted from purification and HTHP, which was consistent with previous studies (Gao et al., 2018; Gornas et al., 2016; Xi et al., 2015).

\subsection{Changes of major compounds of the four extracts}

The calibration curves of C, B1, BCA, EC, EA, rutin, Q3A, K3R, K3G, Q and K were investigated using HPLC-MS (Table S1), and their structural formulae and peaks are shown in Figure S1 and S3, respectively. Based on the HPLC-MS quantitative analysis,
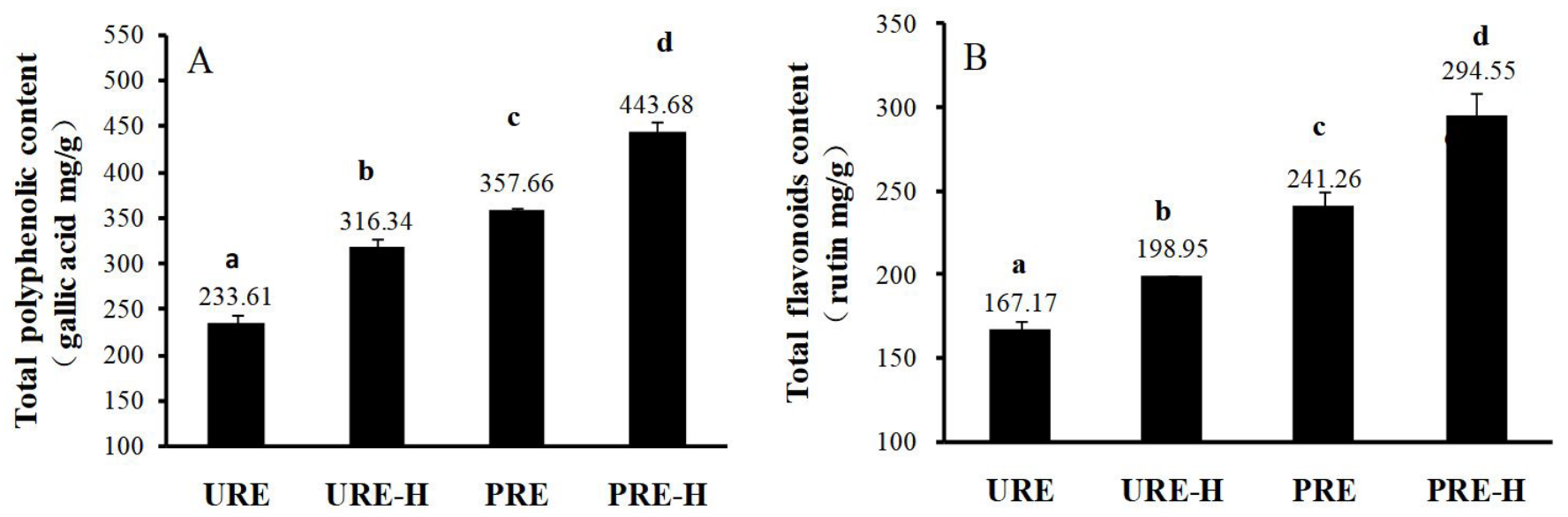

Figure 1. TPC (A) and TFC (B) of the four extracts. Different letters above the columns indicate significantly differences among the extracts $(\mathrm{p}<0.05)$. 

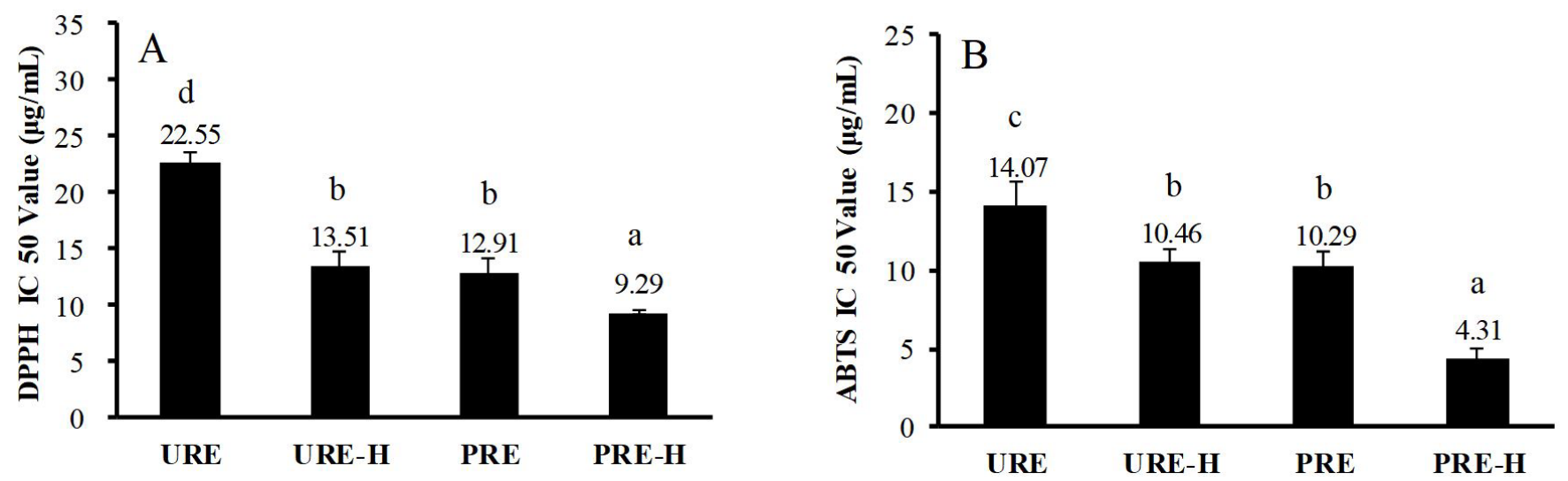

Figure 2. Antioxidant capacity of extracts produced using the DPPH (A) and ABTS (B) methods. Different letters above the columns indicate significantly differences among the extracts $(\mathrm{p}<0.05)$.

Table 1. MICs of the four extracts against three bacteria.

\begin{tabular}{ccccc}
\hline Species & URE & URE-H & PRE & 4 \\
2 \\
E.coli & 8 & 4 & 8 & 4 \\
S.aureus & 16 & 8 & 10 & 8 \\
S.enterica & 20 & 16 & 10 & \\
\hline
\end{tabular}

11 typical polyphenols were identified from the changes in the extract peak areas. Compared with the TPCs and TFCs of the URE, those of the URE-H, PRE and PRE-H increased (Table 2), which indicated that the tested components of raspberry extracts were enhanced after resin purification or HTHP treatment, as demonstrated in the results of the colorimetry (Figure 1). Interestingly, the increases in 11 of the tested polyphenols were different after the two treatments. Using purification through a macroporous resin, all the tested polyphenols of PRE increased by $\sim 30 \%-300 \%$. The highest enrichment rates were for $\mathrm{C}$, B1 and EC, which were all over 100\% (Table 2). However, after the HTHP treatment, the changes in these polyphenols were classified into three groups (Figure S3 and Table 2): 1) Increased; 2) Decreased; and 3) Unchanged.

1) Increased: the EA (Peak 5) accounted for more than $50 \%$ of the TPCs of the four extracts: $73.00 \%(1.2 \mathrm{mg} / \mathrm{g})$ in the URE, $79.22 \%(2.0 \mathrm{mg} / \mathrm{g})$ in the URE-H, $67.00 \%(1.8 \mathrm{mg} / \mathrm{g})$ in the PRE and $74.91 \%(3.2 \mathrm{mg} / \mathrm{g})$ in the PRE-H (Table 2). Thus, its increase after HTHP could be the main factor in the change in the TPCs, which caused the TPC of the PRE to be greater than that of the URE-H, while the TFC of the PRE was less than that of the URE-H. The increase in EA may result from the depolymerization of hydrolyzed tannins to small molecular substances or the loss of glycosides from EA derivatives. EA polymerization products (HHDP-galloyl glucose, Sanguiin $\mathrm{H}-10$ and Sanguiin $\mathrm{H}-6$ ), ellagic acid pentosides and coumarin derivatives (a unit of BCA, belonging to the hydrolyzed tannin after EA polymerization) were identified based on corresponding $m / z(-)$ values in Figure S3 and Table S2, as well as from previous studies in which they were identified in red raspberry (Sójka et al., 2016) and blackberry (Oszmianski et al., 2015). EA is related to the commercial antifungicide fluconazole used on Trichophyton (Li et al., 2015) and, when combined with tetracycline, it inhibits biofilm formation and the associated virulence of Propionibacterium acnes in vitro and in vivo (Sivasankar et al., 2016). Thus, the compounds in these extracts may be important for the treatment of fungal infections and adolescent skin diseases. In addition, the contents of C, B1 and EC (Peaks 1, 3 and 4) of the URE-H and PRE-H were greater than those of the URE and PRE, respectively. C, EC and/or gallocatechin are the basic units in the formation of PAs. The simplest PAs (e.g., B1: C plus EC; Figure 1) and dimers of flavan-3-ol units (Figure S1) have been identified in cocoa and grape seeds (Cádiz-Gurrea et al., 2017). These compounds, in particular B1 and C, in our study, may originate from the developing seeds in unripe raspberry. The antibacterial, antiviral and antifungal activities of $\mathrm{C}$ and $\mathrm{EC}$, the main polyphenols in tea, have received attention in the past. $\mathrm{C}$ can modulate the adherence to oral epithelial cells and inhibit Porphyromonas gingivalis, and also in combination with antibiotics (e.g., metronidazole and tetracycline) (Fournier-Larente et al., 2016) or with curcumin (Betts \& Wareham, 2014), which is a natural polyphenol from Curcuma longa, it has synergistic effects against multidrug-resistant strains of Acinetobacter baumannii. PAs have higher anti-inflammatory and antibacterial effects, and increase dietary health (Cádiz-Gurrea et al., 2017). However, the bioactivity and solubility of compounds depend on their chemical structures. The oligomeric PAs (OPCs) and polymeric PAs (PPAs) remain nonextractable and insoluble, therefore, eluding analysis. They are not usually taken into account in chemical and nutritional studies (Daglia, 2012; Shay et al., 2017). Thus, depolymerized PPAs and OPCs that exist after HTHP may be result in the increases in EC, C, B1 and TFC. 2) Decreased: Four flavonol glycosides (Peak 6, 7, 8 and 9 corresponding to rutin, Q3G, K3R and K3G, respectively) of the URE-H and PRE-H decreased by $\sim 10 \%-20 \%$ after HTHP. In particular, the K3R of the URE decreased from $0.90 \mathrm{mg} / \mathrm{g}$ (53.43\%) before HTHP to 


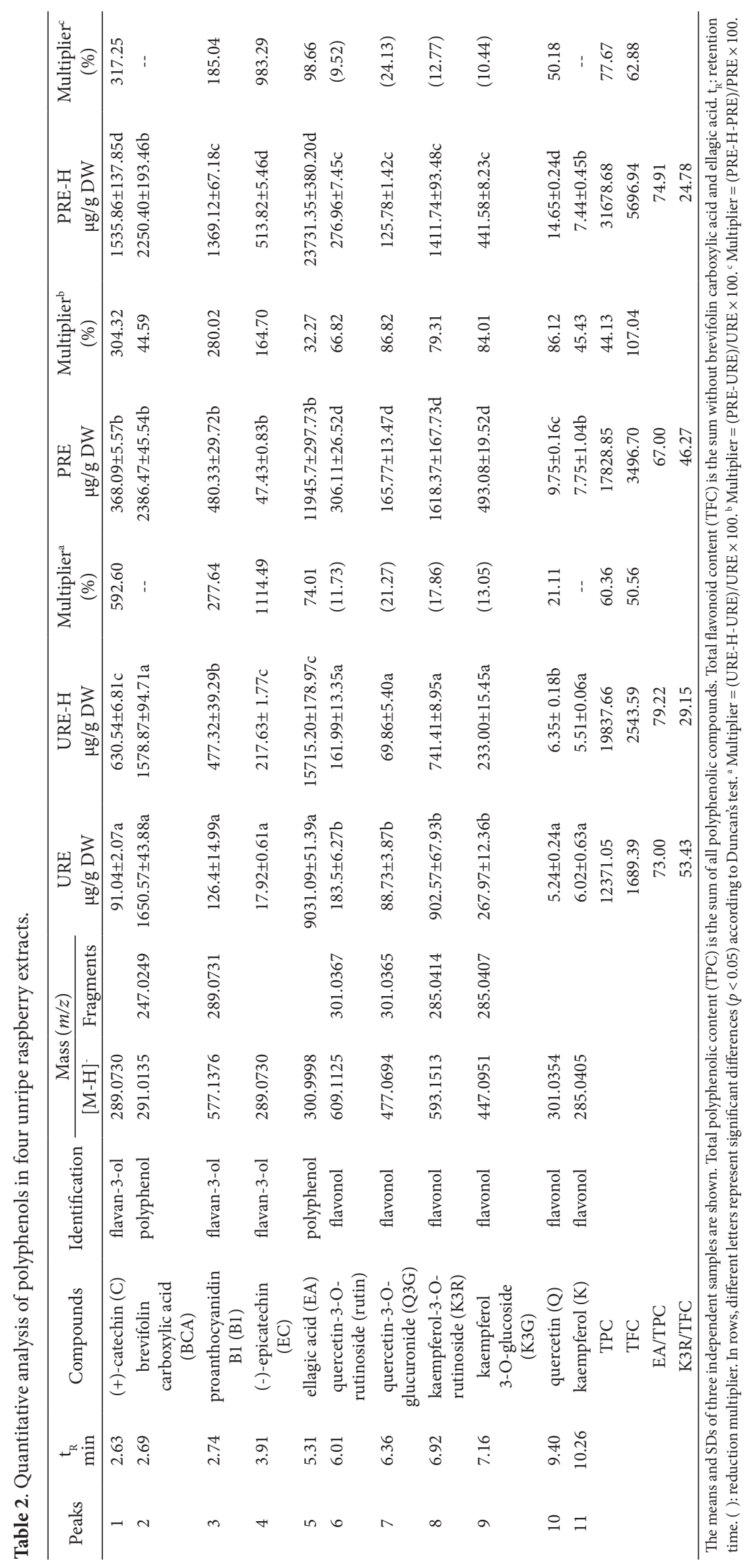


$0.74 \mathrm{mg} / \mathrm{g}(29.15 \%)$ after HTHP, and that of the PRE decreased from $1.62 \mathrm{mg} / \mathrm{g}$ (46.28\%) before HTHP to $1.41 \mathrm{mg} / \mathrm{g}(24.78 \%)$. However, the quercetin (Peak 10) content increased significantly less than the quercetin glycoside decreased. Additionally, kaempferol (Peak 11) decreased because of the reductions in $\mathrm{K} 3 \mathrm{R}$ and $\mathrm{K} 3 \mathrm{G}$. We speculate that both flavonol glycosides (Q3A, K3R, K3G and rutin) and flavonoid aglycones (K and Q) were partially degraded into smaller molecules by HTHP. Similarly, ellagic acid and total phenol levels in muffins enriched with strawberry, sour cherry, and raspberry or black currant pomace were enhanced after high temperature baking $\left(140^{\circ} \mathrm{C}, 180^{\circ} \mathrm{C}\right.$ and $220^{\circ} \mathrm{C}$, respectively), but anthocyanin and flavonol glycoside levels decreased (Gornas et al., 2016). 3) Unchanged: Peak 2 was identified as BCA owing to a deprotonated ion [M-H] ${ }^{-}$at $m / z$ 291 and had the same retention time as the standard reference. Additionally, its fragments ( $m / z$ of 247 and 203) corresponded to losses of carboxylic moieties. BCA has previously been identified in pomegranate (Fischer et al., 2011). The Q and $\mathrm{K}$ contents in the four extracts were very low $(<1 \%$ ) and changed little after HTHP and resin purification. They were not main components of the unripe raspberry extracts.

\section{Conclusion}

In brief, the AC and antibacterial activity of raspberry extracts after HTHP and purification increased along with the TPC and TFC, as assessed by colorimetry and a HPLC-MS analysis. We hypothesized that HTHP may disrupt the forms covalently bound to insoluble polymers and increase the levels of plant polyphenols, in particular, EC, C, EA and B1, but decrease the levels of flavonol glycosides by $\sim 10 \%-20 \%$. Additionally, purification by a macroporous resin is beneficial to the enrichment of most polyphenols. Thus, there is great potential for the bioactive ingredients in the extracts after purification and HTHP. Further investigations that evaluate the correlations between bioactive ingredients and treatments should confirm that HTHP and purification are effective means for preparing extracts.

\section{References}

Benchennouf, A., Grigorakis, S., Loupassaki, S., \& Kokkalou, E. (2017). Phytochemical analysis and antioxidant activity of Lycium barbarum (Goji) cultivated in Greece. Pharmaceutical biology. 55(1), 596-602. https://doi.org/10.1080/13880209.2016.1265987.

Betts, J. W., \& Wareham, D. W. (2014). In vitro activity of curcumin in combination with epigallocatechin gallate (EGCG) versus multidrugresistant Acinetobacter baumannii. BMC Microbiology, 14(172), 1-5. http://dx.doi.org/10.1186/1471-2180-14-172. PMid:24969489.

Cádiz-Gurrea, M. L., Borras-Linares, I., Lozano-Sanchez, J., Joven, J., Fernandez-Arroyo, S., \& Segura-Carretero, A. (2017). Cocoa and grape seed byproducts as a source of antioxidant and antiinflammatory proanthocyanidins. International Journal of Molecular Sciences, 18(376), 1-14. http://dx.doi.org/10.3390/ijms18020376. PMid:28208630.

Daglia, M. (2012). Polyphenols as antimicrobial agents. Current Opinion in Biotechnology, 23(2), 174-181. http://dx.doi.org/10.1016/j. copbio.2011.08.007. PMid:21925860.

Fischer, U. A., Carle, R., \& Kammerer, D. R. (2011). Identification and quantification of phenolic compounds from pomegranate (Punica granatum L.) peel, mesocarp, aril and differently produced juices by HPLC-DAD-ESI/MS(n). Food Chemistry, 127(2), 807-821. http:// dx.doi.org/10.1016/j.foodchem.2010.12.156. PMid:23140740.

Fournier-Larente, J., Morin, M. P., \& Grenier, D. (2016). Green tea catechins potentiate the effect of antibiotics and modulate adherence and gene expression in Porphyromonas gingivalis. Archives of Oral Biology, 65, 35-43. http://dx.doi.org/10.1016/j.archoralbio.2016.01.014. PMid:26849416.

Gao, N., Wang, Y., Jiao, X., Chou, S., Li, E., \& Li, B. (2018). Preparative purification of polyphenols from aronia melanocarpa (chokeberry) with cellular antioxidant and antiproliferative activity. Molecules, 23(1), 1-14. http://dx.doi.org/10.3390/molecules23010139. PMid:29320456.

Goldschmidt, E. E. (2013). The evolution of fruit tree productivity: a review. Economic Botany, 67(1), 51-62. http://dx.doi.org/10.1007/ s12231-012-9219-y. PMid:23538880.

Gornas, P., Juhnevica-Radenkova, K., Radenkovs, V., Misina, I., Pugajeva, I., Soliven, A., \& Seglina, D. (2016). The impact of different baking conditions on the stability of the extractable polyphenols in muffins enriched by strawberry, sour cherry, raspberry or black currant pomace. Lwt - Food Science and Technology, 65, 946-953. http:// dx.doi.org/10.1016/j.lwt.2015.09.029.

Kumar, S., \& Pandey, A. K. (2013). Chemistry and biological activities of flavonoids: an overview. The Scientific World Journal, 2013, 1-16. http://dx.doi.org/10.1155/2013/162750. PMid:24470791.

Li, Z. J., Guo, X., Dawuti, G., \& Aibai, S. (2015). Antifungal activity of ellagic acid in vitro and in vivo. Phytotherapy Research, 29(7), 1019-1025. http://dx.doi.org/10.1002/ptr.5340. PMid:25919446.

Meda, A., Lamien, C. E., Romito, M., Millogo, J., \& Nacoulma, O. G. (2005). Determination of the total phenolic, flavonoid and proline contents in Burkina Fasan honey, as well as their radical scavenging activity. Food Chemistry, 91(3), 571-577. http://dx.doi.org/10.1016/j. foodchem.2004.10.006.

Oszmianski, J., Nowicka, P., Teleszko, M., Wojdylo, A., Cebulak, T., \& Oklejewicz, K. (2015). Analysis of phenolic compounds and antioxidant activity in wild blackberry fruits. International Journal of Molecular Sciences, 16(7), 14540-14553. http://dx.doi.org/10.3390/ ijms160714540. PMid:26132562.

Puupponen-Pimiä, R., Nohynek, L., Meier, C., Kahkonen, M., Heinonen, M., Hopia, A., \& Oksman-Caldentey, K. M. (2001). Antimicrobial properties of phenolic compounds from berries. Journal of Applied Microbiology, 90(4), 494-507. http://dx.doi.org/10.1046/j.13652672.2001.01271.x. PMid:11309059.

Shay, P. E., Trofymow, J. A., \& Constabel, C. P. (2017). An improved butanol- $\mathrm{HCl}$ assay for quantification of water-soluble, acetone: methanol-soluble, and insoluble proanthocyanidins (condensed tannins). Plant Methods, 63(13), 1-11. http://dx.doi.org/10.1186/ s13007-017-0213-3. PMid:28775761.

Silva, S., Costa, E. M., Coelho, M. C., Morais, R. M., \& Pintado, M. E. (2017). Variation of anthocyanins and other major phenolic compounds throughout the ripening of four Portuguese blueberry (Vaccinium corymbosum L) cultivars. Natural Product Research, 31(1), 93-98. http://dx.doi.org/10.1080/14786419.2016.1209668. PMid:27686738.

Sivasankar, C., Maruthupandiyan, S., Balamurugan, K., James, P. B., Krishnan, V., \& Pandian, S. K (2016). A combination of ellagic acid and tetracycline inhibits biofilm formation and the associated virulence of Propionibacterium acnes in vitro and in vivo. Biofouling, 32(4), 397-410. http://dx.doi.org/10.1080/08927014.2016.1148141 . PMid:26930280. 
Sójka, M., Macierzynski, J., Zaweracz, W., \& Buczek, M. (2016). Transfer and mass balance of ellagitannins, anthocyanins, flavan-3-ols, and flavonols during the processing of red raspberries (Rubus idaeus L.) to juice. Journal of Agricultural and Food Chemistry, 64(27), 55495563. http://dx.doi.org/10.1021/acs.jafc.6b01590. PMid:27292440.

Xi, L., Mu, T., \& Sun, H. (2015). Preparative purification of polyphenols from sweet potato (Ipomoea batatas L.) leaves by AB-8 macroporous resins. Food Chemistry, 172, 166-174. http://dx.doi.org/10.1016/j. foodchem.2014.09.039. PMid:25442539.
Zhang, T. T., Lu, C. L., Jiang, J. G., Wang, M., Wang, D. M., \& Zhu, W. (2015a). Bioactivities and extraction optimization of crude polysaccharides from the fruits and leaves of Rubus chingii Hu. Carbohydrate Polymers, 130, 307-315. http://dx.doi.org/10.1016/j. carbpol.2015.05.012. PMid:26076631.

Zhang, T. T., Yang, L., \& Jiang, J. G. (2015b). Bioactive comparison of main components from unripe fruits of Rubus chingii $\mathrm{Hu}$ and identification of the effective component. Food \& Function, 6(7), 2205-2214. http://dx.doi.org/10.1039/C5FO00406C. PMid:26053738. 
Yang et al.

\section{Supplementary Material}

Supplementary material accompanies this paper.

Figure S1. The structural formulas of 11 typical polyphenols in raspberry.

Figure S2. Antibiotic activity of the four extracts. A: E.coli; B: S.aureus; C: S.enterica

Figure S3. HPLC chromatogram of the four extracts.

Table S1. Calibration curves for 11 standard compounds.

Table S2. Qualitative analysis of polyphenols of the four extracts.

This material is available as part of the online article from http://scielo.br/cta 